\title{
Perfil criativo e qualidade de vida: implicações em adultos e idosos com dor lombar crônica
}

\section{Creativeprofileandquality of life implicationsinadutsandeddely with droriclowbadk pain}

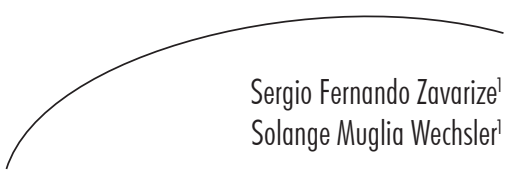

\section{Resumo}

Introdução: A dor lombar é considerada um problema de saúde pública que afeta 70\% a $80 \%$ da população adulta, e a lombalgia, quando crônica, compromete a qualidade de vida em vários níveis. Objetivo: Investigar a relação da criatividade na qualidade de vida em portadores de dor lombar crônica. Método: Amostra composta por 158 participantes, 105 mulheres e 53 homens, de 30 a 88 anos, portadores de dor lombar crônica e diagnóstico de osteoartrose lombar, inscritos em cinco clínicas especializadas. Instrumentos utilizados: Escala do Perfil Criativo; Whoqol-bref; Escala Visual Analógica; Questionário Complementar. Resultados: O coeficiente de Pearson mostrou correlações positivas entre o fator Ousadia com os domínios psicológico e social e do fator Intuição com o físico e ambiental. O fator Individualismo apresentou correlações negativas com os domínios psicológico, social, ambiental e geral. Conclusões: O perfil criativo tem implicações importantes para a qualidade de vida de indivíduos com dor lombar crônica.

\section{Abstract}

Introduction: The low back pain is considered a public health problem and affects $70 \%$ to $80 \%$ of the adult population. When chronic, it undertakes the quality of life on many levels. Objective: To investigate the relations of creative profile in the quality of life of patients with chronic low back pain. Method: Sample of 158 participants, 105 women and 53 men, aged 30 to 88 years, with chronic low back pain, diagnosed with lumbar osteoarthritis, registered in five clinics. Instruments: Scale of Creative Profile; WHOQOL-bref; Visual Analogue Scale; Supplementary Questionnaire. Results: The Pearson coefficient found positive correlations between the factor Daring with the

Palavras-chave: Dor Lombar. Criatividade. Qualidade de Vida. Adulto. Idosos.

Key words: Low Back Pain. Creativity. Quality of Life. Adult. Elderly.

1 Grupo de Pesquisas do Laboratório de Avaliação e Medidas Psicológicas - LAMP. Pontifícia Universidade Católica de Campinas. Campinas, SP, Brasil. 
psychological and social factors, and Intuition with the physical and environmental ones. Individualism factor showed negative correlations with the psychological, social, environmental and general. Conclusions: The creative profile has important implications on the quality of life of individuals with chronic low back pain.

\section{INTRODUÇÃO}

A criatividade pode ser compreendida como um processo no qual existem interações das habilidades cognitivas, características de personalidade e elementos ambientais que facilitam o sujeito a alcançar as descobertas criativas. ${ }^{1,2}$ Portanto, ela se torna fundamental em várias ocasiões da vida, particularmente em momentos difíceis, como na relação com a saúde e com a doença, assim como nas situações de risco. Para os indivíduos criativos, tais circunstâncias são entendidas como desafios a serem vencidos.

Conhecer e identificar as características criativas tem favorecido o desenvolvimento de programas que visam a estimular a criatividade do indivíduo e desenvolver as características de personalidade que estão presentes nas pessoas com alto índice criativo. ${ }^{3}$ Assim, torna-se necessário conhecer as características de personalidade que devem ser incentivadas desde cedo, como forma de facilitar seu desenvolvimento. ${ }^{1}$

Vários autores ${ }^{1-5}$ descreveram as características dos indivíduos criativos, como independência de julgamento, maior tolerância à complexidade, energia elevada, abertura aos impulsos e às fantasias, alto grau de originalidade, intuição, espontaneidade, preferência por situações de risco, alta motivação e curiosidade, elevado senso de humor, confiança em si mesmo, fluência e flexibilidade de ideias, alta sensibilidade externa e interna, inconformismo, abertura a novas experiências, uso elevado de analogias e combinações incomuns, ideias elaboradas e enriquecidas, impulsividade e espontaneidade, autoconceito positivo e sentido de destino criativo., ${ }^{1,6}$

Partindo do pressuposto de que as características criativas impulsionam o indivíduo à solução de problemas e influenciam suas atitudes, sobretudo em situações de risco e dificuldades, pode ser plausível abordar as questões relativas à qualidade de vida do indivíduo em relação ao seu perfil criativo. A criatividade, por conta do seu caráter multidimensional, associando inteligência, personalidade, conhecimento e ambiente social, ${ }^{1,2}$ pode oferecer, por meio de seu processo criativo, instrumentos de transformação, readaptação, ou mesmo de resiliência, em condições de doença ou de dor.

A qualidade de vida, conforme estudos da Organização Mundial da Saúde, é entendida como a "percepção do indivíduo sobre a vida, no contexto da cultura, sistemas e valores ou, ainda, expectativas, padrões e preocupações, relativos a seus objetivos"?

O psiquismo humano, assim como fatores culturais e experienciais, influencia de maneira importante a percepção dolorosa e, consequentemente, a qualidade de vida. Este significado também muda em relação à cultura de determinado grupo social ou raça e, portanto, as causas da dor lombar, em grande parte dos casos, estão relacionadas aos aspectos que influenciam a vida de quem convive com ela, ou seja, há uma ligação estreita entre a doença, a psique e a qualidade de vida. ${ }^{8}$

Por sua vez, componentes emocionais envolvidos no processo de dor crônica podem expressar-se, algumas vezes, de maneira mais significativa que os componentes sensitivos. Emoções e valores simbólicos influenciam a percepção da dor, descaracterizando a proporcionalidade entre estímulo doloroso e a dor percebida pelo indivíduo. Depressão, ansiedade e estresse, associados à mudança de tônus muscular e fatores hormonais, acarretam 
fadiga e, consequentemente, aumento do quadro doloroso. ${ }^{7,9}$ Isto justifica a importância de se investigar, além das questões meramente físicas e objetivas, os aspectos emocionais e as características de personalidade do indivíduo. Neste estudo, foi enfatizado especificamente o perfil criativo.

A lombalgia é considerada uma das alterações musculoesqueléticas mais comuns nas sociedades industrializadas, afetando $70 \%$ a $80 \%$ da população adulta em algum momento da vida, ${ }^{10} \mathrm{ou}$ seja, consiste em um problema de saúde pública. É também uma das principais causas de absenteísmo ao trabalho, além de causar a incapacidade permanente em uma população economicamente ativa e de ser a segunda causa de procura pela assistência médica em doenças crônicas nos Estados Unidos. Configura-se, portanto, como uma das razões mais comuns para aposentadoria por incapacidade total ou parcial. ${ }^{10,11}$

No Brasil, os números não são diferentes do resto do mundo: aproximadamente 10 milhões de brasileiros ficam incapacitados em razão da dor lombar e pelo menos $70 \%$ da população sofrerão uma ocorrência de dor durante a vida. ${ }^{12}$ Esses fatores têm provocado variados temas de pesquisa na área e, portanto, geram a necessidade cada vez maior de investigações referentes à qualidade de vida e ao perfil dos indivíduos acometidos pela doença.

A gênese da lombalgia é influenciada por fatores constitucionais, individuais, posturais e ocupacionais. Entre as causas ocupacionais, destacam-se as sobrecargas na coluna lombar, geradas pelo levantamento de peso excessivo, deslocamento de objetos pesados, permanência numa determinada posição e submetimento a estímulos vibratórios por longos períodos de tempo Da mesma forma, fatores individuais como a altura, o ganho de peso, a obesidade, a postura incorreta, o déficit de força dos músculos abdominais e espinhais e a falta de condicionamento físico são fatores de risco para o aparecimento de dor. Ainda, sinais vegetativos como distúrbios do sono, alterações no apetite e irritabilidade podem estar presentes no quadro apresentado. ${ }^{13}$
Entretanto, algumas condições específicas podem levar à lombalgia, como problemas congênitos, causas degenerativas, processos inflamatórios, infecções, tumores e questões mecânico-posturais. Há ainda a lombalgia mecânico-postural, que representa grande parte da incidência das dores na coluna referidas pela população. Isso ocorre, geralmente, devido a um desequilíbrio entre a carga funcional, que seria o esforço requerido para atividades do trabalho e da vida diária, e a capacidade funcional, que é o potencial de execução para tais atividades. ${ }^{14}$

A classificação dos tipos de dor lombar pode ser organizada de acordo com a duração. As lombalgias agudas apresentam início súbito e duração inferior a seis semanas, enquanto as lombalgias subagudas têm duração de seis a 12 semanas, e as lombalgias crônicas apresentam período maior do que 12 semanas..$^{13}$ Pode-se afirmar que a lombalgia crônica caracteriza-se por uma síndrome incapacitante e por dor, que perdura após o terceiro mês, a contar do primeiro episódio de dor aguda, além da gradativa instalação da incapacidade, muitas vezes tendo início impreciso, com períodos de melhora e piora. ${ }^{13,15}$

Supondo-se que as pessoas criativas podem ter maiores possibilidades de adaptação e reorganização de suas vidas por meio do processo criativo, esperam-se melhores índices de qualidade de vida para esses indivíduos, quando portadores de dor lombar crônica.

O objetivo deste estudo foi realizar a investigação das relações entre a criatividade (perfil criativo), qualidade de vida e a dor lombar crônica em indivíduos adultos e idosos.

\section{MÉTODO}

\section{Participantes}

Esta pesquisa utilizou um enfoque metodológico de abordagem quantitativa. A amostra foi composta por 158 indivíduos portadores de dor lombar crônica, de ambos os sexos (105 mulheres e 53 homens), de 30 a 88 anos de idade, sendo 79 indivíduos com idade entre 30 e 54 anos, e 79 com 55 anos ou mais. 
A média da idade geral da amostra foi de 54,76 anos (desvio padrão de 12,56). A porcentagem de participantes com 60 anos de idade ou mais representou $31 \%$ do total da amostra.

Os participantes foram selecionados por amostragem de conveniência e estavam inscritos para tratamento em três clínicas privadas e duas da rede pública, todas especializadas no tratamento de dor, localizadas em três cidades do interior do Estado de São Paulo. As avaliações foram realizadas nos pacientes na primeira consulta, sem que estes estivessem fazendo uso de medicamentos para o controle da dor lombar.

Como fatores de inclusão, os participantes apresentaram diagnóstico médico de osteoartrose lombar e sintomatologia superior a 12 semanas. A exclusão ocorreu de forma livre, pelo não consentimento do sujeito em participar da pesquisa. Outras situações, como lombalgias decorrentes de gestação ou patologias conjuntas, como pós-operatórios, hérnias de disco, tumores de coluna, problemas de ordem neurológica e uso de medicamentos para controlar a dor, foram da mesma forma consideradas fatores de exclusão para a pesquisa. Foram excluídos 16 dos participantes pré-selecionados.

\section{Instrumentos}

Escala do Perfil Criativo - instrumento validado $^{16}$ com 72 itens compostos de adjetivos descritores da personalidade criativa, extraídos na literatura nacional e internacional, sobre as características da pessoa criativa, agregando cinco perfis de criatividade: Ousadia, Tradicionalismo, Individualismo, Intuição e Prudência. A escala configura-se no formato do tipo Likert de seis pontos (escores de um a seis), na qual o participante assinala uma opção dentre as seis possibilidades de resposta - Discordo Totalmente, DT; Discordo, D; Discordo Parcialmente, DP; Concordo Parcialmente, CP; Concordo, C; Concordo Totalmente, CT. Quanto maior a pontuação na escala, maior a força do perfil criativo. ${ }^{4,5,16}$
O fator Ousadia está relacionado a características positivas para a Criatividade, como fluência, flexibilidade, originalidade, alta sensibilidade externa e interna, espontaneidade, humor, motivação, curiosidade, inconformismo, abertura a novas experiências, situação de risco e confiança em si mesmo, além de representar características voltadas à confiança na própria capacidade, na busca de autonomia e motivação por mudança.

O fator Tradicionalismo indica características como evitar riscos, insegurança em dar opiniões, evitar discutir ideias, seguir padrões predeterminados, o que se configura como fator oposto à criatividade.

O Individualismo relaciona-se a condições opostas às características criativas, como egoísmo, preocupação com necessidades pessoais, falta de humanitarismo, dificuldade em aceitar ideias diferentes das suas e preferência em trabalhar sozinho.

O fator Intuição aponta para a facilidade em fantasiar, brincar com ideias, desenvolver atividades com prazer e emoção. Apresenta itens ligados a alta sensibilidade interna, fantasia, imaginação e impulsividade, que descrevem positivamente a pessoa criativa.

A Prudência se revela por atenção nos processos de resolução de problemas, atribuindo detalhes, planejando e organizando informações - características da mesma forma presentes na personalidade criativa. Os fatores opostos à criatividade são considerados importantes para o estudo do perfil criativo e são carregados negativamente para as avaliações dos resultados. Os dados psicométricos deste instrumento foram avaliados por Reis e apresentaram consistência interna, validade e precisão. ${ }^{16}$

Escala Visual Analógica (E.V.A.) instrumento válido e amplamente utilizado, que avalia a percepção da intensidade da dor e consiste de uma linha reta cujo extremo esquerdo aponta para a "ausência de dor" e o extremo direito para a "pior dor possível", na qual os valores 
variam de zero a dez; o paciente é instruído para se autoavaliar, marcando o ponto que indique a intensidade da sua dor. ${ }^{17} \mathrm{~A}$ consistência interna não pode ser calculada porque a medida consiste em apenas um item, mas escalas de itens simples, assim como a E.V.A., são válidas e extremamente sensíveis para mudanças no estado do paciente.

Instrumento de Avaliação da Qualidade de Vida (WHOQOL-bref), da Organização Mundial da Saúde (OMS) - The World Health Organization Quality of Life Assessment - bref Whoqol-bref. Traduzido e validado para o Brasil, consiste em uma escala tipo Likert de cinco itens onde o participante aponta uma opção entre cinco possibilidades. É composto por 26 questões, duas gerais sobre qualidade de vida, e as demais representando quatro domínios: físico, psicológico, social e meio ambiente. As características psicométricas do WHOQOLbref preencheram todos os critérios de consistência interna, validade discriminante, validade concorrente, validade de conteúdo e confiabilidade teste-reteste. ${ }^{18}$

Questionário Complementar - Foi desenvolvido para este trabalho pelo pesquisador; contém quatro questões que incluem perguntas sobre a frequência de atividades de lazer, condições de melhora e piora dos quadros de dor, e se o participante empregou ações no sentido de aliviar ou tentar esquecer a dor. Este instrumento teve a finalidade de enriquecer os dados da pesquisa junto à população estudada.

\section{Procedimentos}

Depois de aprovada a pesquisa pelo Comitê de Ética em Pesquisa com Seres Humanos, foi enviada uma carta de autorização para diretores das clínicas especializadas em tratamento de dor e/ou de coluna. Em seguida, os participantes receberam o termo de consentimento livre e esclarecido para sua anuência em participar da pesquisa.

Cada sujeito preencheu os testes individualmente e de próprio punho, sob o acompanhamento e orientação do pesquisador. Isto ocorreu no momento da primeira consulta e, portanto, os participantes não faziam uso de medicamentos para controle da dor.

A média dos resultados da Escala Visual Analógica (E.V.A) permitiu a categorização da dor em dois níveis: 1 para os participantes que apresentaram escore de zero a sete (inclusive), e 2 para os resultados que estavam acima de sete até dez.

Foram, da mesma forma, categorizadas as faixas etárias de 30 a 54 anos e acima dos 54 anos. Optou-se por este tipo de categorização por sua representatividade na amostra estudada. Desta forma, procurou-se analisar tanto os indivíduos adultos como os mais maduros, o que dividiu proporcionalmente os participantes. Dentro da categorização dos indivíduos de 54 anos de idade ou acima, a porcentagem de participantes com 60 anos de idade ou mais, isto é, os idosos, representou $62 \%$. Já para o total da amostra, este número apontou para $31 \%$ dos participantes.

A partir da categorização da dor lombar e da faixa etária em dois níveis na amostra, estes resultados foram relacionados para as análises estatísticas com os dados da Escala do Perfil Criativo. Do mesmo modo, foram considerados os resultados do instrumento de Avaliação da Qualidade de Vida, Whoqol-bref, em cada domínio. Foram ainda utilizados os dados colhidos por meio do Questionário Complementar, observando-se a frequência das ações referentes a lazer, estratégias de alívio e de distração da dor.

Para as análises estatísticas, utilizou-se o programa Statistical Package for the Social Sciences (SPSS), versão 18.0. Procedeu-se, então, às análises dos dados, utilizando a Análise da Variância Multivariada (MANOVA) para comparar os efeitos de sexo, idade e níveis de dor (E.V.A.) com os domínios de qualidade de vida e (perfis) de criatividade. O sexo foi considerado uma variável fixa; idade e dor, covariáveis. Os domínios de qualidade de vida e fatores do perfil criativo foram considerados variáveis dependentes. Para este tipo de análise, 
a MANOVA representa a variação entre médias amostrais e a variação entre os indivíduos dentro da amostra, verificada por meio do teste "F".

Além disso, foi utilizada a correlação de Pearson para investigar as relações entre os fatores do perfil criativo e os domínios de qualidade de vida e, ainda, para as relações entre as estratégias de lazer, alívio e distração da dor com o perfil criativo, utilizando-se o total bruto das amostras para cada instrumento. Esperou-se atingir um nível de significância $5 \%$, isto é, com $p \leq 0,05$ entre as correlações a serem observadas.

Este trabalho foi aprovado pelo Comitê de Ética em Pesquisa com Seres Humanos da PUCCampinas, sob Protocolo no 852/09, e a pesquisa cumpriu todos os princípios éticos contidos na Declaração de Helsinki, além de atender à legislação pertinente.

\section{RESULTADOS}

A porcentagem dos participantes que procuraram o tratamento para a dor lombar crônica foi maior para o sexo feminino, correspondendo a $66,5 \%$ da amostra, contra $33,5 \%$ para o sexo masculino.

Quanto à percepção da dor, avaliada por meio da E.V.A. em relação ao sexo, pode-se observar que as mulheres apresentaram níveis mais elevados de dor, em que o sexo feminino apresentou média de 7,42 pontos, enquanto no masculino os valores foram de 6,40 para a percepção da dor. O teste $t$ de Student apresentou diferença significativa entre os $\operatorname{sexos}(t=2,657 ; \mathrm{p} \leq 0,01)$.

A figura 1 exibe os resultados da escala E.V.A. de dor em relação ao sexo, por meio do gráfico tipo boxplot.

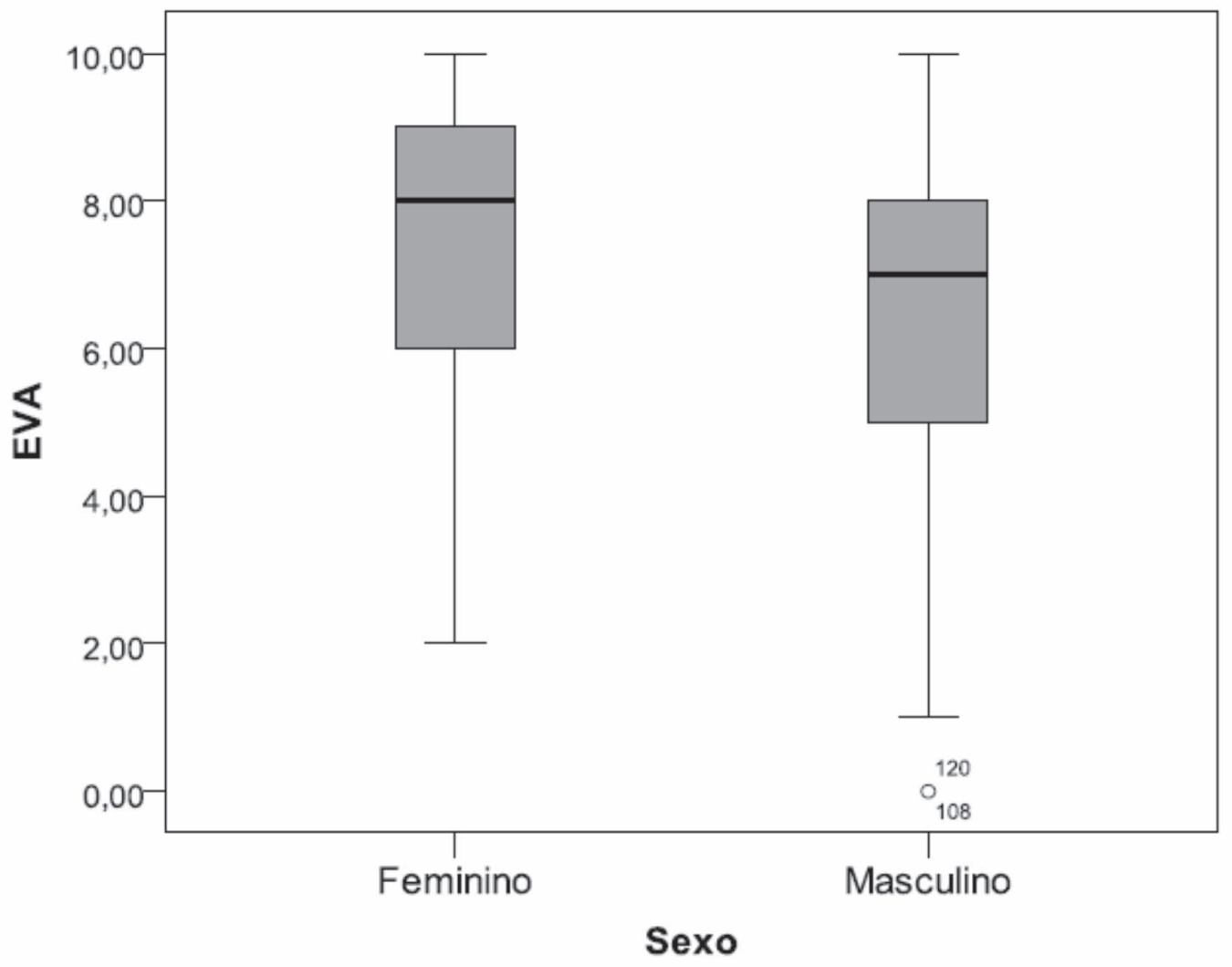

Figura 1 - Distribuição dos resultados da escala E.V.A. para avaliação da dor em relação ao sexo. 
Os resultados relacionados à escala de dor apresentados na figura 1 mostraram uma mediana de valor 8,0 para o sexo feminino e 7,0 para o masculino, com uma distribuição dos valores, da mesma forma, mais elevados para o sexo feminino. A moda (resultado mais frequente) teve os valores iguais aos da média $(7,0)$.

Tabela 1 - Média dos resultados da escala E.V.A. por faixa etária. Campinas, SP, 2011.

\begin{tabular}{lccc}
\hline \multicolumn{1}{c}{ Faixa Etária } & N & EVA & Desvio Padrão \\
\hline 30 a 54 anos & 79 & 6,87 & 2,31 \\
55 anos ou mais & 79 & 7,29 & 2,34 \\
Geral & 158 & 7,08 & 2,33 \\
\hline
\end{tabular}

A tabela 1 apresenta a média dos resultados da escala E.V.A. segundo a faixa etária. Portanto, o nível médio de dor foi 6,87 para o grupo de 30 a 54 anos, e 7,29 para indivíduos com mais de 54 anos de idade, o que mostra um aumento nos índices de dor para faixa etária mais elevada.
Aestatísticautilizada foia Análise Multivariada de Variância (MANOVA), verificada por meio do teste $\mathrm{F}$, que representa a variação entre médias amostrais e variação entre os indivíduos dentro da amostra.

Tabela 2 - Análise multivariada de variância nos domínios de qualidade de vida de acordo com o sexo, idade e E.V.A. Campinas, SP, 2011.

\begin{tabular}{lccc}
\hline & \multicolumn{3}{c}{ Valores de F } \\
\cline { 2 - 4 } \multicolumn{1}{c}{$\begin{array}{c}\text { Domínios de } \\
\text { Qualidade de Vida }\end{array}$} & Sexo & Idade & EVA \\
\hline Domínio 1 Físico & 0,100 & $6,350^{* *}$ & $75,034^{* * *}$ \\
Domínio 2 Psicológico & 0,899 & 0,176 & $16,920^{* * *}$ \\
Domínio 3 Social & 0,000 & 0,647 & $14,426^{* * *}$ \\
Domínio 4 Ambiental & 1,922 & 0,778 & $32,648^{* * *}$ \\
Domínio 5 Global & 0,149 & 0,000 & $36,933^{* * *}$ \\
\hline
\end{tabular}

Nota. ${ }^{*} \mathrm{p} \leq 0,05 ; * * \mathrm{p} \leq 0,01 ; * * \mathrm{p} \leq 0,001$

A tabela 2 apresenta a MANOVA nos domínios de qualidade de vida de acordo com o sexo, idade e E.V.A. Como pode ser observado, existiu relação significativa entre idade e qualidade de vida para o domínio físico. Já os resultados de E.V.A. se relacionaram significativamente com todos os domínios do construto. 
Tabela 3 - Análise multivariada de variância nos fatores de criatividade de acordo com sexo, idade e E.V.A. Campinas, SP, 2011.

\begin{tabular}{lccc}
\hline & \multicolumn{3}{c}{ Valores de F } \\
\cline { 2 - 4 } Fatores de Criatividade & Sexo & Idade & EVA \\
\hline Fator 1 Ousadia & 0,150 & $7,511^{* *}$ & 0,838 \\
Fator 2 Tradicionalismo & 0,960 & $20,820^{* * *}$ & $6,423^{* *}$ \\
Fator 3 Individualismo & $5,321^{*}$ & $17,996^{* * *}$ & $8,671^{* *}$ \\
Fator 4 Intuição & 0,140 & $4,594^{*}$ & 3,512 \\
Fator 5 Prudência & 1,537 & 0,067 & 0,421 \\
\hline
\end{tabular}

Nota. ${ }^{*} \mathrm{p} \leq 0,05 ; * * \mathrm{p} \leq 0,01 ; * * * \mathrm{p} \leq 0,001$

Da mesma forma que a tabela 1 , a tabela 3 apresentou os resultados da Análise Multivariada da Variância (MANOVA) entre sujeitos nos fatores de Criatividade em relação ao sexo, idade e E.V.A., indicando efeitos significativos para o fator Individualismo em relação ao sexo; Ousadia, Tradicionalismo, Individualismo e Intuição em relação à idade. Da mesma forma, Tradicionalismo e Individualismo obtiveram resultados significativos em relação à dor. Os fatores Tradicionalismo e Individualismo apresentam características opostas à personalidade criativa, isto é, são fatores negativos para a criatividade.

Em relação ao Questionário Complementar, nos dados que se referiram à frequência na utilização de estratégias para alívio da dor, foram observados como procedimentos mais utilizados pelos participantes: tomar medicação, fazer repouso, usar compressas quentes, corrigir a postura, fazer alongamentos, fazer exercícios, fazer massagem, dentre outros.

Nas respostas relacionadas à distração da dor, os participantes muitas vezes se confundiram com atividades de lazer, o que se repetiu nas respostas. Encontraram-se como respostas mais frequentes: distrair-se pensando em outras coisas, conversar, fazer caminhada, assistir televisão, ir à igreja, passear, ler, dentre outros.

Para a frequência de atividades de lazer, observaram-se como mais utilizadas as seguintes atividades: assistir televisão, passear, ler, ficar com a família, visitar amigos, ir à igreja, visitar familiares, dentre outras atividades. $O$ sexo feminino apresentou médias mais elevadas para os índices relacionados às atividades de lazer e de distração da dor. 
Tabela 4 - Correlação de Pearson entre os fatores da Escala do Perfil Criativo e os domínios do instrumento de qualidade de vida. Campinas, SP, 2011.

\begin{tabular}{|c|c|c|c|c|c|c|c|c|c|c|}
\hline & $\begin{array}{c}\text { Fator } \\
1\end{array}$ & $\begin{array}{c}\text { Fator } \\
2\end{array}$ & $\begin{array}{c}\text { Fator } \\
3\end{array}$ & $\begin{array}{c}\text { Fator } \\
4\end{array}$ & $\begin{array}{c}\text { Fator } \\
5\end{array}$ & $\begin{array}{c}\text { Domínio } \\
1\end{array}$ & $\begin{array}{c}\text { Domínio } \\
2\end{array}$ & $\begin{array}{c}\text { Domínio } \\
3\end{array}$ & $\begin{array}{c}\text { Domínio } \\
4\end{array}$ & $\begin{array}{c}\text { Domínio } \\
\text { Geral }\end{array}$ \\
\hline Fator 1 Ousadia & - & & & & & & & & & \\
\hline Fator 2 Tradicionalismo & $0,177^{*}$ & - & & & & & & & & \\
\hline Fator 3 Individualismo & $-0,332^{* *}$ & $-0,382 * *$ & - & & & & & & & \\
\hline Fator 4 Intuição & $0,319 * *$ & $0,160^{*}$ & $-0,026$ & - & & & & & & \\
\hline Fator 5 Prudência & 0,127 & $-0,166^{*}$ & $-0,029$ & $-0,020$ & - & & & & & \\
\hline Domínio 1 Físico & 0,155 & 0,033 & $-0,156$ & $0,202^{*}$ & 0,002 & - & & & & \\
\hline Domínio 2 Psicológico & $0,313 * *$ & 0,133 & $-0,341^{* *}$ & 0,076 & $-0,046$ & $0,628^{* *}$ & - & & & \\
\hline Domínio 3 Social & $0,202^{*}$ & 0,050 & $-0,158^{*}$ & 0,078 & $-0,045$ & $0,398 * *$ & $0,490^{* *}$ & - & & \\
\hline Domínio 4 Ambiental & 0,140 & 0,093 & $-0,315^{* *}$ & $0,162^{*}$ & $-0,031$ & $0,562^{* *}$ & $0,559 * *$ & $0,369 * *$ & - & \\
\hline Domínio Geral & 0,078 & 0,088 & $-0,191 *$ & 0,140 & 0,037 & $0,612^{* *}$ & $0,560 * *$ & $0,363^{* *}$ & 0,541 ** & - \\
\hline
\end{tabular}

Nota. ${ }^{*} \mathrm{p} \leq 0,05 ;{ }^{* *} \mathrm{p} \leq 0,01 ; * * * \mathrm{p} \leq 0,001$.

Os resultados da tabela 4 mostraram correlações fortes e significativamente positivas entre o fator Ousadia e os domínios psicológico e social, apontando, desta forma, implicações para a qualidade de vida desses indivíduos. O fator Individualismo apresentou fortes correlações significativamente negativas com os domínios psicológico, social, ambiental e geral. Já o fator Intuição mostrou correlações significativamente positivas com o domínio físico e ambiental. Os fatores Tradicionalismo e Prudência não apresentaram correlação significativa com os domínios de qualidade de vida.

\section{DISCUSSÃO}

Até então, não havia na literatura nacional e internacional pesquisas que procurassem relacionar o perfil criativo à qualidade de vida de indivíduos tanto adultos como idosos, portadores de lombalgia crônica. Os achados deste trabalho apontaram para resultados importantes para o conhecimento que engloba as três áreas estudadas.

A porcentagem dos participantes que procuraram o tratamento para a dor lombar crônica foi bem maior para o sexo feminino, correspondendo a 66,5\% da amostra, contra $33,5 \%$ para o sexo masculino, corroborando os estudos de Ponte ${ }^{19}$ e Almeida et al. ${ }^{20}$ Observou-se, ainda, que o sexo feminino procurou os serviços de tratamento (clínicas especializadas) em maior número, e obteve índices significativamente mais elevados de percepção dolorosa que o sexo masculino.

De uma maneira geral, os níveis álgicos encontrados na amostra foram elevados para ambos os sexos, o que se justifica pelo fato de a aplicação do instrumento de avaliação da dor ter ocorrido no momento da primeira consulta ou avaliação, isto é, quando nenhum tratamento havia sido iniciado.

O significado da dor para homens e mulheres pode ainda ser influenciado por questões culturais e sociais que acabam por permitir à mulher uma expressão ou manifestação mais aberta ou de aceite da dor, enquanto se encorajam os homens a subvalorizá-la. Para o homem, a segurança diante da dor pode representar, de certa forma, sua virilidade. Portanto, essas questões podem e devem ser consideradas como favorecedoras tanto da forma de expressão, como da queixa de dor entre as mulheres. ${ }^{21}$ 
Os resultados mostraram que o domínio físico de qualidade de vida - questões como dor e desconforto, energia, fadiga, sono e repouso - foi afetado pela idade e pela dor (E.V.A.). Em indivíduos de faixa etária mais elevada, há um consequente decréscimo nas condições físicas, sobretudo em indivíduos portadores de problemas em coluna, o que justificaria esses resultados. Além disso, estudos comprovam que indivíduos com mais de 60 anos se queixam duas vezes mais que indivíduos com menos de 60 anos de idade. ${ }^{22}$

Observou-se ainda, nos resultados apresentados, que todos os domínios de qualidade de vida foram afetados pela dor. Este fato apontou a dor como um agente importante de incapacitação e limitação funcional, afetando os domínios físicos, psicológicos, sociais e em relação ao meio ambiente.

Em relação aos resultados do perfil criativo, encontraram-se efeitos significativos no fator Individualismo para idade, sexo e dor; no fator Tradicionalismo para idade e dor; e nos fatores Ousadia e Intuição para idade.

Em relação aos efeitos observados do perfil criativo para a idade, notaram-se resultados significativos para os fatores Ousadia, Tradicionalismo, Individualismo e Intuição. É importante lembrar que, em casos de pacientes com dor crônica, o aumento da idade e o tempo de ocorrência dos eventos levam a uma redução nos reforços positivos disponíveis, ocasionando desânimo e depressão. Portanto, o fato de os escores das médias da criatividade terem se apresentado mais baixos na faixa etária mais elevada pode se justificar pela própria cronicidade da doença, que induz ao tempo prolongado da dor, a fatores físicos incapacitantes, ao estresse e à tendência à depressão. Este fato pode levar o indivíduo idoso a se perceber de forma negativa, generalizando seus pensamentos depressivos e determinando uma alta morbidade psicológica nesses quadros. ${ }^{8,23,24}$
Para os resultados dos fatores como Ousadia, Intuição e Prudência, que são característicos da pessoacriativa, estes não apresentaram, pela Análise da Variância Multivariada, efeitos significativos em relação à dor. Já os fatores Tradicionalismo e Individualismo, que contemplam características contrárias à criatividade, estes sim, apresentaram efeitos significativos para a dor. Este fato pode estar relacionado ao sentido de que as pessoas criativas percebem a dor de maneira diferente ou ainda menos intensa. Portanto, estes resultados parecem indicar uma melhor maneira, por parte das pessoas criativas, de lidarem com o problema de forma a amenizar seus efeitos em relação às pessoas com características opostas à criatividade.

Os participantes desta pesquisa apresentaram atividades de lazer em grande número, como alternativas para lidar com o problema da dor lombar crônica. Estratégias de alívio da dor foram da mesma forma frequentes, além de estratégias de distração da dor. O sexo feminino apresentou maior frequência de estratégias de lazer e de distração da dor que o masculino.

No tocante aos resultados dos construtos do perfil criativo e de qualidade de vida, a correlação de Pearson mostrou diversas analogias existentes entre eles. Comparando-se os fatores do perfil criativo com os domínios de qualidade de vida, foram encontradas correlações significativamente positivas entre o fator Ousadia com os domínios psicológico e social, e do fator Intuição com o físico e ambiental. Já o fator Individualismo apresentou correlações significativamente negativas com os domínios psicológico, social, ambiental e geral.

Os fatores Ousadia e Intuição apresentaram diversas características marcantes da personalidade criativa. Ousadia está relacionada a características como fluência, flexibilidade, originalidade, alta sensibilidade externa e interna, espontaneidade, humor, motivação, curiosidade, inconformismo, abertura a novas experiências, situação de risco e confiança em si mesmo; já a Intuição, a facilidade em fantasiar, brincar com ideias, desenvolver atividades com prazer e emoção., ${ }^{1,5}$ 
O fator Prudência, embora fosse composto de características importantes da personalidade criativa, não apresentou resultados significativos com a qualidade de vida, provavelmente por ser o fator mais fraco do instrumento, apresentando apenas seis itens.

Como proposta para futuros estudos, sugere-se que, além dos aspectos analisados nesta pesquisa, outras variáveis, como altura, obesidade, atitudes posturais e demais componentes emocionais envolvidos no processo de dor crônica sejam investigados.

\section{CONCLUSÕES}

A Escala do Perfil Criativo apresenta cinco fatores que foram investigados nesta pesquisa. Destes cinco, três se relacionaram significativamente com a qualidade de vida: Ousadia, Individualismo e Intuição. E, embora os fatores Tradicionalismo e Prudência não tenham apresentado resultados significativos, indicaram tendências com a qualidade de vida,

\section{REFERÊNCIAS}

1. Wechsler SM. Criatividade: descobrindo e encorajando. Campinas: Impressão Digital do Brasil, 2008.

2. Nakano TC, Wechsler SM. Figural creativity path from high school to university. Bol. psicol. 2006; 56(125):205-19.

3. Amaral Filho J. Cultura, Criatividade e Desenvolvimento. Políticas Culturais em Revista. 2009;1(2):4-19.

4. Nakano TC, Wechsler SM. Criatividade: Características da produção científica brasileira. Aval. psicol. 2007; 6(2):261-70.

5. Wechsler SM. Estilos de pensar e criar: impacto nas áreas educacional e profissional. Rev. Psicodebate. 2008; 7:207-18.

6. Alencar EMLS, Fleith DS. Contribuições teóricas recentes ao estudo da criatividade. Psicologia: Teoria e Pesquisa. 2003;19(1):1-8.

7. Pereira RJ, Cotta RMM, Franceschini SCC, Ribeiro RCL, Sampaio RF, Priore, SE, et al Contribuição dos domínios físico, social, psicológico e ambiental para e futuros estudos poderão investigar melhor esta questão. Portanto, os resultados demonstraram implicações importantes do perfil criativo na qualidade de vida dos sujeitos avaliados. Os participantes que apresentaram maiores índices de criatividade pela Escala do Perfil Criativo exibiram melhores níveis nos domínios de qualidade de vida. Apresentaram, ainda, maior número de estratégias de distração e de atividades de lazer.

Esses resultados devem favorecer a elaboração de novas formas de tratamento e intervenção em todas as faixas etárias. Um modelo a ser sugerido para a inclusão da criatividade nesse contexto seria a partir de uma atuação interdisciplinar, onde ao mesmo tempo em que se desenvolvessem os tratamentos clínicos convencionais, se estimulassem o desenvolvimento de atividades criativas, laborais (ocupacionais) e de expressão corporal (corporeidade), o que poderia ocorrer no formato de oficinas. Este modelo poderia proporcionar aos indivíduos de diferentes faixas etárias, não apenas a melhora nos sintomas, mas também de sua qualidade de vida. a qualidade de vida global de idosos. Rev. psiquiatria Rio Grande do Sul. 2006;28(1):27-38.

8. Almeida FF, Costa Junior AL, Doca FNP, Turra V. Experiência de Dor e Variáveis Psicossociais: o Estado da Arte no Brasil. Temas em Psicologia. 2010;18(2):367-376

9. Lin, T.Y., Stump, P., Kaziyama, H.H.S., Teixeira, M.J., Imamura, M., Greve, J.M.A. Medicina física e reabilitação em doentes com dor crônica. Rev. Med. (São Paulo), 80(ed. esp. pt.2):245-55, 2001.

10. Andrade SC, Araujo AGR, Vilar MJP. Escola de Coluna: revisão histórica e sua aplicação na lombalgia crônica. Rev. Bras. Reumatologia [Periódicos na Internet]. 2005 [acesso em: 24 jun 2011];45(4):224-8. Disponível em: http://www. scielo.br/scielo.php?script=sci_arttext\&pid $=$ S048200042005000400006\&lng=en\&nrm=iso.

11. Briganó JU, Macedo CSG. Análise da mobilidade lombar e influência da terapia manual e cinesioterapia na lombalgia. Semina: Ciências Biológicas e da Saúde. 2005;26(2):75-82 
12. Toscano JJO, Egypto EP. A influência do sedentarismo na prevalência de lombalgia. Rev. Bras. Medicina do Esporte. 2001;7(4):132-7.

13. Iamamura ST, Kaziyama HHS, Iamamura M. Lombalgia. Medicina. 2001;80(2):375-90.

14. Souza MC, Vasconcelos LC, Arnal TC, Carvalho G, Miyamoto ST. Deep Water Running no tratamento da lombalgia mecânico-postural: relato de caso. Rev. Neuroc. 2008;16(1):62-6.

15. Silva MC, Fassa AG, Valle NCJ. Dor lombar crônica em uma população adulta do Sul do Brasil: prevalência e fatores associados. Cad. de Saúde Pública. 2004;20(2):377-85.

16. Reis CL. Escala de Perfil Criativo Profissional: validade e precisão de instrumento. Tese de Doutorado não publicada. Pontifícia Universidade Católica de Campinas. Campinas, (SP), 2005.

17. Bryce TN, Budh CN, Cardenas DD, Dijkers M, Felix ER, Finnerup NB, et al. Pain After Spinal Cord Injury: An Evidence-based Review for Clinical Practice and Research. J. Spinal Cord. Med. 2007;30(5):421-40.

18. Fleck MP, Louzada S, Xavier M, Chachamovich, E, Vieira G, Santos L, et al. Aplicação da versão em português do instrumento abreviado de avaliação da qualidade de vida "WHOQOL-bref". Rev Saúde
Pública. [Periódicos na Internet]. 2000 [acesso em: 02 mai 2009]; 34(2):178-83. Disponível em: http://www. scielosp.org/scielo.php?script=sci_arttext\&pid=S003489102000000200012\&lng=en\&nrm=iso.

19. Ponte C. Lombalgia em cuidados de saúde primários: sua relação com características sócio-demográficas. Rev Port Clinica Geral. 2005;21:259-67.

20. Almeida ICGB, Sá KN, Silva M, Batista A, Matos MA, Lessa I. Prevalência de dor lombar crônica na população da cidade de Salvador. Rev Bras Ortopedia. 2008;43(3):96-102.

21. Kreling MCGD, Cruz DALM, Pimenta CAM. Prevalência de dor crônica em adultos. Rev. Bras. Enfermagem. 2006;59(4):509-13.

22. Andrade FA. Pereira LV, Souza FAEF. Mensuração da dor no idoso: uma revisão. Rev. Latino-Am. Enfermagem. 2006;14(2):271-6.

23. Cruz RM, Sarda Junior JJ. Diagnóstico de aspectos emocionais associados à lombalgia e à lombociática. Aval. psicol. [Periódico da internet]. 2003 [acesso em: 03 jul 2011]; 2(1):29-33. Disponível em: http://pepsic. bvsalud.org/scielo.php?script=sci_arttext\&pid=S167704712003000100004\&lng $=$ pt\&nrm $=$ iso.

24. Abreu AM, Faria CDCM, Cardoso SMV, Salmela LFT. Versão brasileira do Fear Avoidance Beliefs Questionnaire. Cad. Saúde Pública. 2008;24(3):615-23. 